\title{
Patrones para la organización del conocimiento en los Sistemas de Información Curricular. Un caso de estudio
}

\author{
Soleidy Rivero Amador * \\ Maidelyn Díaz Pérez ** \\ María José López Huertas Pérez *** \\ Dayron Armas Peñas ****
}

Artículo recibido: 6denoviembrede2013.

Artículo aceptado: 9 de marzo de 2015.

\section{Resumen}

La historia de la humanidad delata que la imperiosa necesidad de sistematizar todos los conocimientos sobre el mundo exterior ha sido una de las causas fundamentales de la aparición de las clasificaciones del conocimiento científico. En la actualidad, el desarrollo y evolución de los sistemas de gestión de información y conocimiento han logrado representar bastante bien la complejidad de la organización del conocimiento, a pesar de la naturaleza desarrolladora del propio conocimiento que se clasifica, y sus características sistémicas y esencia interdisciplinar. El objetivo principal de esta investigación es el análisis de la clasificación del

* Universidad de Pinar del Río. Pinar del Río, Cuba. soly@upr.edu.cu

** GrupodeGestióndelnformaciónyConocimiento(PROGINTEC).Universidadde PinardelRío"HermanosSaízMontesdeOca",Cuba.maidelyn@upr.edu.cu *** Universidad de Granada, España.mjlopez@ugr.es

**** GrupodeGestióndelnformaciónyConocimiento(PROGINTEC).Universidadde Pinar del Río“Hermanos Saíz Montes de Oca”, Cuba.dayron@upr.edu.cu INVESTIGACIÓN BIBLIOTECOLÓGICA,Vol.30,Núm.68,enero/abril,2016,México,ISSN: 0187-358X. pp. 91-107 
conocimiento procedente de los resultados científicos de los proyectos de investigación para identificar patrones en su composición que permitan su gestión. Se toman como caso de estudio los profesores investigadores que desarrollan proyectos científicos en la Universidad de Pinar del Río y se propone como instrumentos el Sistema de Gestión de Información y Conocimiento Institucional de la Universidad de Pinar del Río con enfoque curricular, así como otras técnicas de diagnóstico para la identificación de patrones en la estructuración del conocimiento de la institución.

Palabras clave: Organización del conocimiento; Sistemas de información curricular; Taxonomías del conocimiento científico.

\section{Abstract}

A case study of knowledge organization patterns within Curricular Information Systems

Soleidy Rivero-Amador, Maidelyn Díaz-Pérez, María José López-Huertas Pérez and Dayron Armas-Peñas

History teaches us that need to systematize knowledge of the outside world is the main driver in the creation of classification systems of scientific knowledge. The development and evolution of the prevailing systems of information and knowledge management have achieved a respectable representation of the complexity of the organization of knowledge, even in the face of the unfolding nature of the knowledge classified, its systemic features and interdisciplinary essence. This research aims principally to provide an analysis of the classification of knowledge of scientific research results and identify compositional patterns that may serve to enhance its management. The paper consists of a case study of scientific researchers of the University of Pinar del Río, and employs the curricular approach entailed in the Institutional Information and Knowledge Management System of the University of Pinar del Rio, as well as other diagnostic techniques to identify patterns in the structuring of the institution's knowledge.

Keywords: Knowledge Organization; Information Systems Curriculum; Taxonomies of Scientific Knowledge. 


\section{Introducción}

L a organización del conocimiento es la ciencia de estructurar sistemáticamente grupos de unidades de conocimiento (conceptos) de acuerdo con sus características inherentes (Dalhberg, 2006). Los postulados formulados en la década de 1990 expresan que la organización del conocimiento se inclina hacia puntos de vista sociales e interpretativos; tal es el caso del análisis del discurso, los estudios de género y el análisis de dominio. Desde ese entonces se han desarrollado varios enfoques semióticos y crítico-hermenéuticos (Hjørland, 2005). El objetivo principal de esta ciencia es aplicar operaciones como la clasificación y la ordenación para crear sistemas de distribución física e intelectual que respeten la secuencialidad, la jerarquía, la asociatividad y otros aspectos que resulten de interés para garantizar un acceso adecuado a la información y al conocimiento desde el nivel institucional.

El propio desarrollo del proceso de investigación científica es un ejemplo consecuente de la complejidad de la organización del conocimiento. Es evidente que los nuevos problemas y fenómenos científicos son irreductibles a una estricta visión disciplinaria. De igual modo, el enfrentamiento a la complejidad de la realidad actual debe realizarse a partir de un cambio de visión o perspectiva científico-metodológica. La apertura es necesaria y la interacción puede proporcionar un mayor enriquecimiento de la percepción científica de la realidad (Morin, 1995). Siempre que el conocimiento se clasifique y organice se deberá tener en cuenta su carácter dinámico, en especial en el desarrollo y aplicación de los Sistemas de Información en el ambiente organizacional.

Pese a los grandes esfuerzos realizados por los Organismos de Ciencia y Tecnología (OCYT), persisten problemas que comparten la mayoría de las instituciones (Navarrete y Banqueri, 2008):

- Coexistencia de diferentes instrumentos de captura de datos que recogen la misma información y no tienen sistemas adecuados de normalización, almacenamiento y recuperación.

- Ineficientescapacidades de integración, interoperabilidadycomunicación entre las bases de datos para tal efecto, y poca homogeneización en los formatos de currículos de los investigadores.

- Poco aprovechamiento y utilización de la información que se sistematiza en los organismos responsables de la gestión de la ciencia y la tecnología, tanto para su gestión estratégica y proyección de políticas como para promover e incrementar la visibilidad de sus resultados. Esto incide en escasos intercambio, colaboración y comunicación entre 
científicos e investigadores en los ámbitos regional, nacional e internacional.

Muchos de estos problemas se han solucionado con la implantación de Sistemas de Información Científica (SIC) ajustados a las características de cada organización o región, si bien aún hay ciertos problemas con los enfoques y alcances de estos sistemas. Esta problemática se refleja en las insuficiencias en la gestión de la actividad científica tecnológica y sus resultados, su imposibilidad para estructurar el conocimiento científico que posee una institución y medir de forma eficiente el impacto de la ciencia como proceso social (Armas, Díaz y Giraldes, 2008; Báez et al., 2008).

El presente trabajo aborda parte de estas problemáticas pero se orienta específicamente al análisis de la estructuración del conocimiento de una organización y su representación mediante diferentes herramientas para estructurar lo que sabe una organización mediante las áreas del conocimiento que abordan sus investigaciones. El objetivo principal es analizar la clasificación del conocimiento procedente de los resultados científicos de los proyectos de investigación para identificar patrones en su composición que permitan su gestión. Se utilizan como caso de estudio los profesores investigadores que desarrollan proyectos científicos en la Universidad de Pinar del Río y se propone como herramienta el Sistema de Gestión de Información y Conocimiento Institucional de la Universidad de Pinar del Río (CV-UPR) con enfoque curricular, así como otras técnicas de diagnóstico para la identificación de patrones en la estructuración del conocimiento de dicha institución.

\section{Materiales y métodos}

El CV-UPR se diseñó e implementó desde hace varios años en la Universidad de Pinar del Río (UPR), está registrado y certificado y ha sido validado en otros estudios. En este sistema, el curriculum vitae (CV) del investigador constituye la única y principal fuente de entrada y actualización de datos. Una de sus principales características es que fue desarrollado para la actividad investigativa, pues se diseñó una estructura jerárquica que abarca toda la actividad y su composición, incluyendo diferentes taxonomías para clasificar resultados científicos (Armas, Díaz y Giraldes, 2008). Para el análisis documental fueron consultados los informes de la Vicerrectoría de Investigación, Informatización y Postgrado (VRIIP), así como sus principales documentos metodológicos y los Balances de la Ciencia y la Técnica en el periodo que se analiza. 
Con el fin de complementar este estudio se utilizaron técnicas empíricas que permiten obtener información cuantitativa en fuentes no documentales. Se aplicó un diagnóstico a la población objeto de estudio, conformada por investigadores pertenecientes a la UPR que tienen la responsabilidad de coordinar proyectos de investigación, y se estudiaron los proyectos de investigación del periodo 2011-2013 que estén vigentes o en ejecución. El diagnóstico declaró una población de 33 investigadores que coordinaron proyectos en el periodo analizado. Se determinó aplicar el cuestionario a la totalidad de la población sin realizar muestreo. En el procesamiento de los datos se utilizaron los programas Statistical Package for Social Science (SPSS, versión 11.5, 2004) y Microsoft Excel (2010) para lograr una mejor interpretación y vinculación de los datos.

\section{Resultados}

De manera general, la ciencia es considerada como un sistema de producción de información, en particular en forma de publicaciones. La publicación es considerada como cualquier "información registrada en formatos permanentes y disponibles para el uso común" (Spinak, 1998: 142). Esta información registrada puede estar no sólo en las publicaciones seriadas y de impacto, sino también en los procesos cotidianos de la ciencia, a nivel institucional y en todo el quehacer del investigador como protagonista de este proceso.

El proceso de gestión de la ciencia y la técnica es el encargado de definir y evaluar las políticas para su desarrollo a nivel nacional o regional. Para cumplir con este propósito, los responsables de estas actividades necesitan del diseño de metodologías que guíen tanto la recolección de la información como el uso de sistemas de información que faciliten el análisis y proporcionen aproximaciones sobre las capacidades y dinámicas científicas.

El diseño de estos sistemas está encaminado a dar respuesta a necesidades concretas de la gestión operacional de los organismos o instituciones de fomento a la investigación y desarrollo tecnológico, a niveles micro, meso y macro. En el ámbito institucional, los SIC surgen para cumplimentar los siguientes objetivos (Armas, Díaz y Giraldes, 2008):

- Estructurar la información operativa y funcional que requieren las organizaciones para operar eficientemente y alcanzar resultados.

- Responder a las necesidades concretas de las organizaciones que fomentan la investigación científica y tecnológica para el desarrollo sostenible de la innovación y el cambio tecnológico. 
- Desarrollar y apoyar a nivel macro las políticas científicas y tecnológicas que necesitan las organizaciones y sus procesos.

- Definir y evaluar las estrategias seguidas en el desarrollo de las actividades de investigación, desarrollo e innovación (1+D+i).

- Evaluar la producción y actividad científico-tecnológica de sus investigadores e instituciones.

- Administrar adecuadamente la distribución de los recursos materiales y humanos dedicados a la I+D+i.

- Fomentar la colaboración, el intercambio, la transferencia científico-tecnológica y la innovación en ámbitos nacionales e internacionales.

Con estos propósitos se refleja claramente la interacción interna y externa en los SI. Las actividades de un investigador y sus interacciones con el resto de los actores de la ciencia ocurren en un entorno institucional, por lo que es desde este punto de vista que se lograrán mayores resultados a nivel regional en la implementación de indicadores para la gestión de la ciencia y la técnica.

Los CV de los investigadores proporcionan información muy valiosa sobre tareas adicionales que forman parte de los resultados de las investigaciones. La observación de la actividad que rodea a un determinado investigador permite

valorar el CV de los científicos y la encuesta como fuentes de información. El primero como conjunto de datos para la obtención de indicadores de la actividad científica y la segunda como fuente de información para establecer [...] el marco social (estructura organizativa del entorno, capital humano disponible, etc.) y económico (financiación de la investigación) en el que los científicos desarrollan habitualmente su actividad. (Martín-Sempere y Rey-Rocha, 2009:2)

En la literatura empírica se muestran trabajos que utilizan el CV como fuente de información para estudiar los comportamientos de la ciencia en sus distintos niveles de actuación. Estos estudios se realizan con el objetivo de valorar el impacto de la afiliación de los miembros de los centros de investigación sobre la productividad, la colaboración y las subvenciones (Gaughan, Branco y Bozeman, 2007). Para el investigador, el CV es una representación de su "valor de conocimiento" (Jaramillo, Lopera y Albán, 2008).

La UPR reconoce tanto la importancia del CV de cada profesor investigador como las potencialidades de uso de los sistemas de información curricular para gestionar la actividad científico-tecnológica a nivel institucional, por lo cual diseñó e implementó el Sistema de Gestión de Información y Conocimiento Institucional de la Universidad de Pinar del Río (CV-UPR). Entre sus principales objetivos está la necesidad de conocer el comportamiento de 
la actividad científica en todas las áreas para su gestión, evaluación y proyección, la identificación de patrones investigativos y la estructuración de todo el conocimiento que posee la universidad según las taxonomías existentes a nivel nacional e internacional.

En determinadas partes del CV se actualizan los tipos de resultados, las áreas del conocimiento en las que se obtienen y los tipos de salida que se materializan en diferentes publicaciones. A continuación se detallan los campos del CV en los que el investigador actualiza los resultados de su labor:

- Titulación académica de posgrado: resultados relacionados con las investigaciones que realizó para obtener sus títulos de posgrado.

- Tesis asistidas: resultados relacionados con sus asesorías de tesis, ya sea en el pregrado o en el posgrado.

- Resultados de investigación asociados a proyectos: relacionados con el trabajo del investigador en el proyecto de investigación al cual esté vinculado.

- Resultados de investigación no asociados a proyectos, relevantes para el investigador y la institución.

Con esta estructura se profundiza en los resultados del proceso de investigación de cada investigador y de la institución, generados o no dentro de un proyecto de investigación. Los diferentes resultados (metodología, producto, proceso, etc.) generan a su vez un conjunto amplio de tipologías de salida: artículos, patentes, libros, monografías, registros de productos informáticos y no informáticos, marcas, normas, etcétera.

En la estructura del CV-UPR cada salida de resultado incluye diferentes áreas del conocimiento; en este caso, el profesor investigador clasifica cada uno de sus resultados según tres taxonomías: la Taxonomía de la Organización de las Naciones Unidas para la Educación, la Ciencia y la Cultura (Unesco), la Taxonomía de la Organización para la Cooperación y el Desarrollo Económico (OCDE) y la clasificación cubana, vigente en ese país. La Taxonomía de la Unesco es de carácter internacional y la Taxonomía de la OCDE es muy utilizada en Hispanoamérica.

La Figura 1 representa la estructura en campos de la parte del CV-UPR orientada a colectar datos provenientes de investigaciones: tipos de resultados, áreas del conocimiento y salidas del resultado. Esta distribución permite medir el conocimiento que se generó por cada tipo de actividad investigativa y estructurar los resultados de la investigación en función de la actividad que los originó, según la naturaleza del propio conocimiento. 


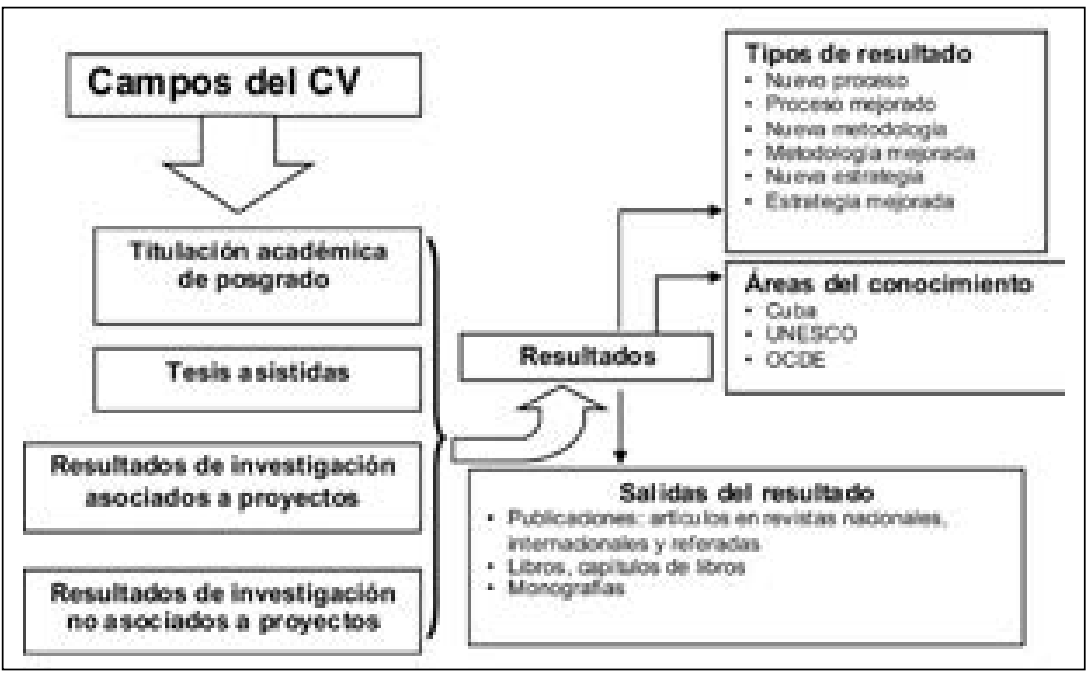

Figura 1. Campos del CV que se actualizan con resultados de las investigaciones Fuente: elaboración propia con base en Armas, Díaz y Giraldes, 2008

Esta distribución dentro del sistema CV-UPR ha permitido conocer la composición disciplinar de las investigaciones en la universidad. Se infiere que existe una propensión a establecer relaciones interdisciplinares en diferentes áreas de la UPR; para constatarlo se propone realizar un diagnóstico a los resultados que provienen de proyectos de investigación.

Los resultados de las técnicas empleadas constataron un amplio grupo de investigaciones interdisciplinares cuyos resultados provienen de distintas áreas y disciplinas del conocimiento, por lo que en varios casos se dificulta su clasificación en las diferentes taxonomías. Prevalecen también efectos de interdisciplinariedad de la ciencia en tesis de doctorado. Este comportamiento amerita un estudio posterior acerca de las características de los proyectos de investigación de la institución y de las líneas de investigación e investigadores implicados.

Los coordinadores de proyectos afirman en un $80 \%$ que los resultados de los proyectos que coordinan están relacionados con más de una disciplina científica (Figura 2) (pág. s.).

Al cruzar dos de las variables que se investigan, el $37 \%$ de los encuestados afirma que su proyecto tiene de 5 a 10 miembros y está relacionado con más de una disciplina científica (Figura 3) (pág. s.). 


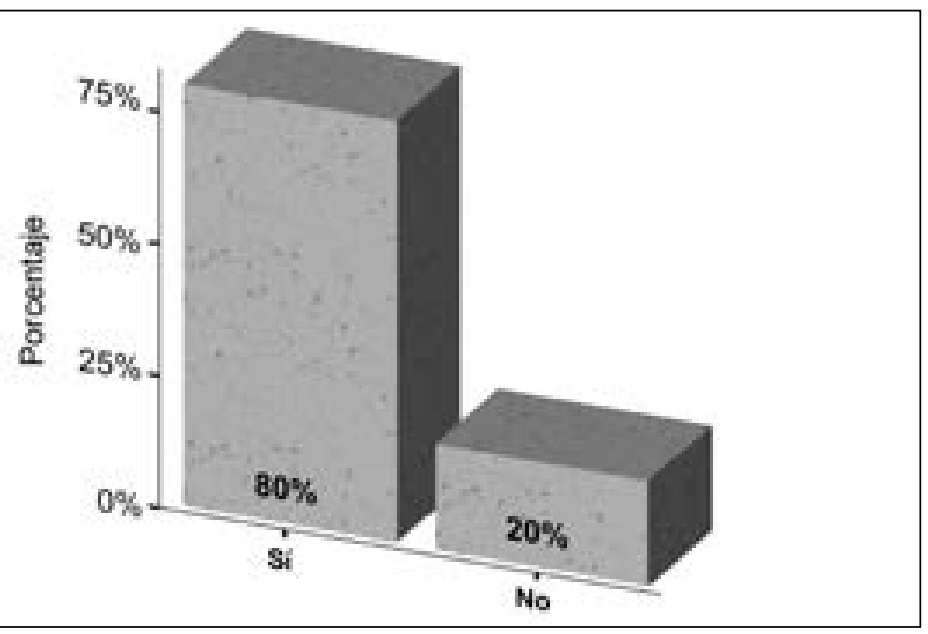

Figura 2. Respuesta de la pregunta 5 del cuestionario Fuente: elaboración propia utilizando el SPSS

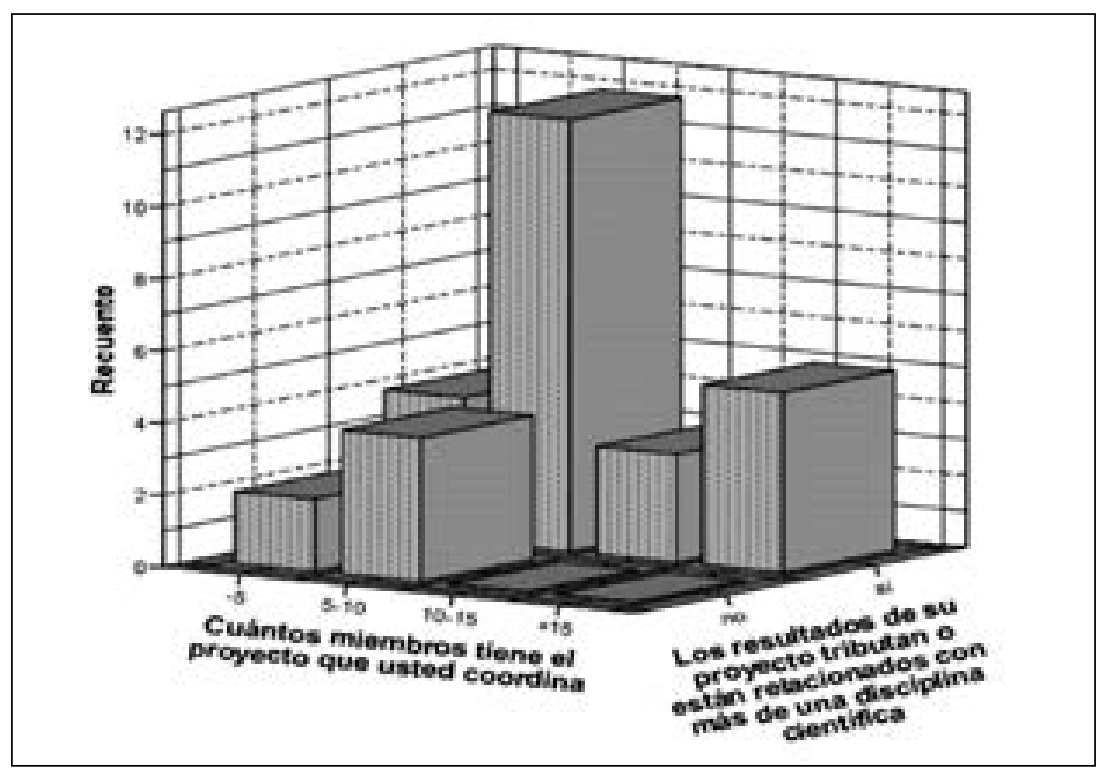

Figura 3. Comparación matricial de las respuestas a dos preguntas Fuente: elaboración propia utilizando el SPSS

De forma similar, quienes coordinan proyectos con más de 15 miembros afirman tener resultados relacionados con varias disciplinas científicas. Este análisis evidencia que hay ciertas características de los proyectos analizados 
que pueden influir en la determinación de relaciones interdisciplinares. Esta es una de las razones que influye en la clasificación de los resultados, siendo difícil acotarlos a un área determinada.

La cantidad de miembros del proyecto, el objetivo que persigue, su alcance y su nivel de interacción con varias disciplinas para solucionar un determinado problema de la realidad constituyen factores que influyen en el grado de interdisciplinariedad. Las áreas relacionadas con las ciencias sociales, por lo general, tienen un comportamiento interdisciplinar diferente.

Se pudo constatar que, de las 61 líneas de investigación de la UPR en el periodo analizado, 30 trabajan en conjunto o necesitan del aporte de otras áreas de la ciencia. Entre ellas destacan Ciencias Pedagógicas, Ciencias Forestales, Ciencias Económicas y Ciencias Técnicas (Figura 4). Las combinaciones más frecuentes son las siguientes:

- Matemáticas

- Ciencias Sociales Aplicadas, Matemáticas, Ciencias Económicas, Ciencias Agrarias

- Ciencias Sociales Aplicadas, Matemáticas, Ciencias Económicas

- Matemáticas, Ciencias Económicas, Ciencias Agrarias

- Ciencias Biológicas

- Ciencias Económicas, Matemáticas

- Química

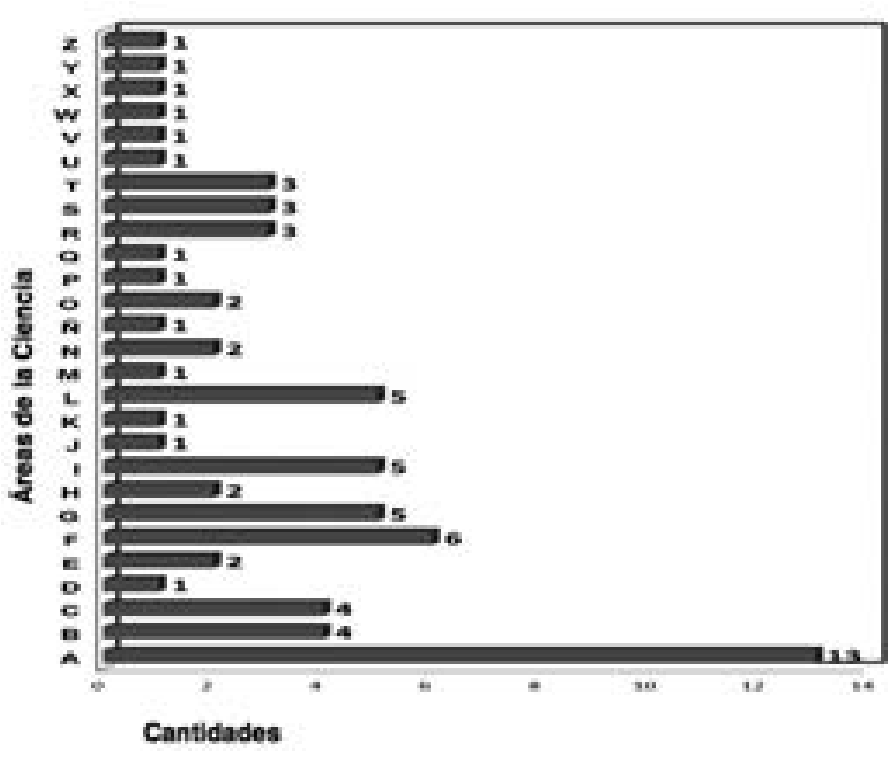




\begin{tabular}{|c|l|}
\hline \multicolumn{1}{|c|}{ Leyenda } \\
\hline A & Matemáticas \\
\hline B & $\begin{array}{l}\text { CienciasSocialesAplicadas,Matemáticas,CienciasEconómicas,Ciencias } \\
\text { Agrarias }\end{array}$ \\
\hline C & Ciencias Sociales Aplicadas, Matemáticas, Ciencias Económicas \\
\hline D & Ciencias Técnicas \\
\hline E & Matemática y Química \\
\hline F & Matemáticas, Ciencias Económicas, Ciencias Agrarias \\
\hline G & Ciencias Biológicas \\
\hline H & Matemáticas, Ciencias Económicas, Química e Informática \\
\hline I & Ciencias Económicas y Matemáticas \\
\hline J & Sociología \\
\hline K & Matemáticas,Sociología,CienciasAgrarias,Química,Biología,Psicología \\
\hline L & Química \\
\hline M & Ciencias Económicas, Ciencias Políticas y Matemáticas \\
\hline N & Matemáticas, Ciencias Agrarias e Informática \\
\hline$\tilde{N}$ & Ciencias Sociales Aplicadas, Matemáticas, Geología, Biología \\
\hline O & Ciencias Agrónomas y Biología \\
\hline P & Sociología, Psicología, Ciencias Económicas \\
\hline Q & Filosofía y Ciencias Sociales Aplicadas \\
\hline R & Ciencias Sociales Aplicadas \\
\hline S & Ciencias Agrarias \\
\hline T & Ciencias de la Educación \\
\hline U & Matemáticas y Ciencias Técnicas \\
\hline V & Geología Regional y Yacimientos Minerales \\
\hline W & Ciencias Económicas \\
\hline X & Psicología y Teoría de la Comunicación \\
\hline Y & Matemática y Geoquímica \\
\hline Z & Física \\
\hline & \\
\hline
\end{tabular}

Figura 4. Áreas de la ciencia que aportan resultados a las tesis doctorales Fuente: elaboración propia utilizando el SPSS

Matemáticas es un ejemplo representativo en la Figura 5 (pág. s.), ya que es un área que aporta métodos necesarios para otras investigaciones, al igual que la Estadística. Se deriva que las investigaciones interdisciplinares son aquellas con mayores combinaciones de áreas relacionadas con sus resultados. Cada investigación doctoral, a su vez, responde a una línea de investigación determinada, lo que manifiesta la existencia de varias líneas de investigación en la institución que se nutren de diversas áreas de las ciencias. 


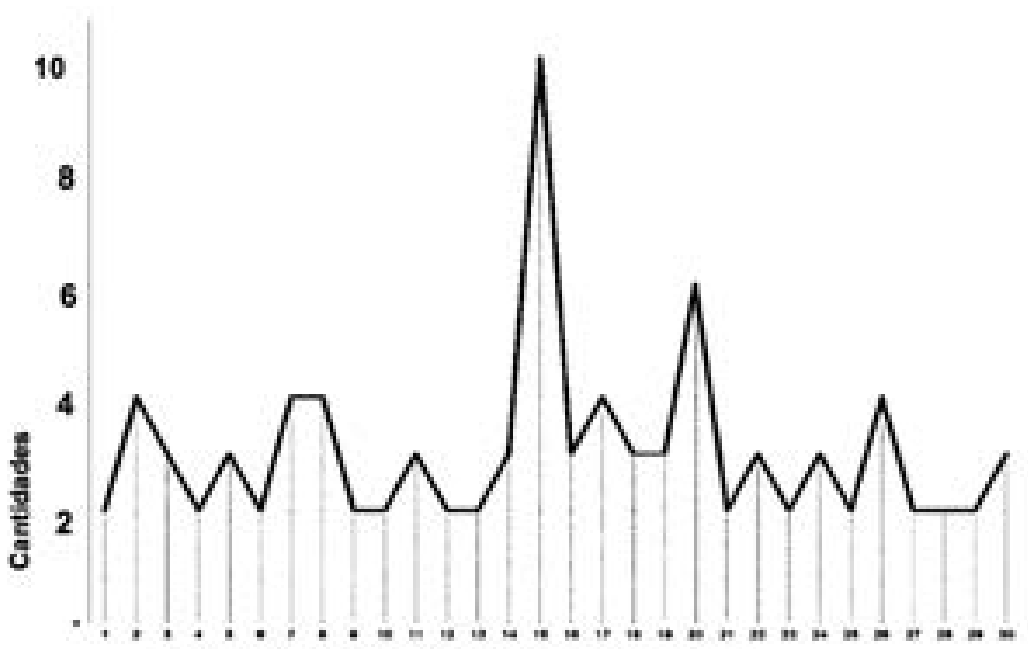

Lineas de Imvestigación de las tesis doctorales

\begin{tabular}{|l|l|}
\hline \multicolumn{2}{|l|}{ Leyenda } \\
\hline 1 & Patentometría \\
\hline 2 & Cooperativismo \\
\hline 3 & Gestión del Turismo Rural \\
\hline 4 & Hidrogeología \\
\hline 5 & Construcción de Caminos Forestales \\
\hline 6 & Manejo Integrado de Plagas \\
\hline 7 & Tecnología de la Madera \\
\hline 8 & Vinculación Universidad-Empresa \\
\hline 9 & Informatización de Procesos Contables \\
\hline 10 & GestiónEconómicoFinancieradeProyectosdelnnovaciónTecnológica \\
\hline 11 & Procesos Termoquímicos \\
\hline 12 & Sistema de Comercialización en la Enseñanza Superior \\
\hline 13 & Administración y Gestión de Empresas \\
\hline 14 & Economía Internacional \\
\hline 15 & Pedagogía y Didáctica de la Educación Superior \\
\hline 16 & Pago por Servicios Ambientales \\
\hline 17 & Gestión de Recursos Humanos \\
\hline 18 & Teoría Económica de la Transición al Socialismo \\
\hline 19 & Silvicultura \\
\hline 20 & Ecosistemas Costeros y Educación Ambiental \\
\hline 21 & Agroecología del Sistema Forestal \\
\hline 22 & Desarrollo Social Comunitario y Educación Popular \\
\hline 23 & Pirólisis y Gasificación en Lecho Fluidizado de Residuos Sólidos \\
\hline 24 & OrientaciónPsicopedagógicadeestudiantesenlaNuevaUniversidadCuba- \\
\hline & na \\
\hline
\end{tabular}




\begin{tabular}{|l|l|}
\hline 25 & Geoquímica de las Rocas y Minas \\
\hline 26 & Lingüística \\
\hline 27 & Linguodidáctica \\
\hline 28 & Estudios prelexicográficos y preterminográficos \\
\hline 29 & Intrusión Marina, Calidad de Agua y Geología Ambiental \\
\hline 30 & Inventario Forestal \\
\hline
\end{tabular}

Figura 5. Cantidad de áreas del conocimiento que aportan resultados a la investigación Fuente: elaboración propia utilizando el SPSS

Las líneas relacionadas con mayor cantidad de áreas de las ciencias son Pedagogía y Didáctica de la Educación Superior, Ecosistemas Costeros y Educación Ambiental (Figura 5). Estas dos líneas se relacionan respectivamente con 10 y 6 áreas de las ciencias. Existen 5 líneas de investigación que se relacionan con 4 áreas de las ciencias: Cooperativismo, Tecnología de la Madera, Vinculación Universidad-Empresa, Gestión de Recursos Humanos y Lingüística.

\section{Discusión de los resultados}

Tal como afirman Hjørland y Albrechtsen (1995), en el complejo escenario de la organización del conocimiento el mejor modo de enfocar su aplicación a la realidad de los Sistemas de Información es estudiando cómo piensan las personas e imitar esas regularidades del pensamiento. En el caso de estudio analizado se cumple esta afirmación al contrastar los resultados de las investigaciones en los proyectos de investigación con la clasificación en las taxonomías que utiliza el sistema CV-UPR.

Se corrobora que no existen formas únicas de organizar el conocimiento resultante del proceso de investigación de la institución, es decir, las taxonomías existentes no son absolutas. En el caso de la información proveniente de resultados de proyectos de investigación es preciso diseñar representaciones que faciliten la organización del conocimiento con sus propias peculiaridades, a partir de diferentes criterios o posturas, tales como la relevancia del tema o línea de investigación, las conexiones existentes entre las diferentes disciplinas (de una misma área o de distintas), las demandas de los resultados de los proyectos hacia otras áreas de la ciencia y la cantidad y diversidad de los miembros de los proyectos.

Es evidente que el aspecto social ejerce una notable influencia en el proceso de organización del conocimiento, con la influencia de ideologías, tradiciones y paradigmas, combinados con el aspecto el intelectual. Lo primordial es lograr viabilizar el uso del conocimiento por parte de sus usuarios, reales o potenciales (Hjørland, 2003; Peña, 2010). 
Con base en lo anterior, se propone que la clasificación de los resultados investigativos se realice a partir de las líneas de investigación a las que pertenece cada trabajo, puesto que los responsables de proyectos de investigación, los jefes de departamentos y los responsables de centros de estudio y grupos de investigación poseen mayor capacidad para clasificar estas líneas en los diferentes campos y disciplinas de las taxonomías utilizadas para clarificar el conocimiento científico.

Esta propuesta relaciona directamente las líneas de investigación con las investigaciones que tributan a ellas. Las líneas aparecerían clasificadas en las taxonomías utilizadas por el sistema CV-UPR y el investigador debe clasificarse en la línea a la que pertenece; esto es sencillo para el investigador pues forma parte de su trabajo cotidiano. Los responsables de clasificar las líneas de investigación son los jefes de proyectos, los jefes de departamentos, los responsables de centros de estudio y grupos de investigación y otros responsables de líneas de investigación de la institución; todos ellos deben ser capacitados al respecto. De este modo se facilita la clasificación y organización del conocimiento para su estructuración en el sistema CV-UPR.

El análisis cuenta con aspectos tanto positivos como desfavorables; ambos se enlistan a continuación.

Aspectos positivos:

- Existe integración en el desarrollo de proyectos de investigación entre especialistas pertenecientes a distintas áreas de la institución y fuera de ella.

- Los clientes de los proyectos trabajan líneas de investigación relacionadas con la actividad investigativa de la universidad.

- Los coordinadores de proyectos conocen el fenómeno de la interdisciplinariedad de la ciencia y lo relacionan con perfiles y líneas de investigación que trabajan en los diferentes proyectos que dirigen.

Aspectos desfavorables:

- Los investigadores confunden el campo con la disciplina científica cuando van a clasificar sus resultados.

- La información sobre ciencia y técnica en la UPR se encuentra muy dispersa en las diferentes áreas de la institución. La fuente principal es el propio investigador, por lo que se necesita o bien su presencia o la posibilidad de consultarlo constantemente.

- El procesamiento y análisis de los resultados y desempeño de los proyectos de investigación se convierte en un proceso engorroso para las 
personas encargadas de coordinar los proyectos y para las autoridades institucionales.

Por último, un aspecto que vale la pena destacar es que la mayor cantidad de resultados provenientesdelosproyectos deinvestigaciónestán relacionadoscon el área de la Ciencia de la Educación. En la clasificación de los investigadores los mayores porcentajes están en campos que se relacionan con esta actividad, según las tres taxonomías utilizadas. Se pudo corroborar que existe total armonía entre los criterios de los investigadores analizados y la información proveniente de los informes de balance de investigaciones de la UPR en igual periodo.

Con esta propuesta se realiza una organización del conocimiento desde una concepción plural que conjuga lo cognitivo, lo social y lo tecnológico, que permite conocer todo el proceso en su extensión de forma participativa. En este caso se respeta la organización lógico-semántica y discursiva que los propios investigadores han usado para construir y organizar sus conocimientos (García, 2001), y se realiza la organización del conocimiento de forma más armónica dentro del sistema de información, uno de los paradigmas principales dentro del proceso de investigación científica (Smiraglia, 2012).

\section{Conclusiones}

El estudio de la bibliografía científica, en correspondencia con las técnicas empíricas utilizadas, mostró la validez que tiene la inclusión de la organización del conocimiento resultante de las investigaciones en el sistema de información científica de una institución.

La clasificación de los resultados de investigación debe ser estructurada mediante las taxonomías del conocimiento científico que existen a nivel nacional e internacional.

Las categorías del conocimiento o áreas de la ciencia establecen a nivel internacional una clasificación del conocimiento de manera disciplinar; este comportamiento en ocasiones desfavorece el proceso de categorización de los resultados de la ciencia, ya que cada vez son más influenciados por la combinación de disciplinas y el surgimiento de nuevas subdisciplinas interrelacionadas.

El diagnóstico realizado a investigadores responsables de proyectos de investigación muestra que la práctica de clasificar los resultados por áreas del conocimiento no es habitual ni es exigida por las autoridades. Se recomienda incorporar este proceder a partir del surgimiento e incremento de proyectos que articulan diferentes saberes y obtienen diferentes resultados interdisciplinares. 
Se propone la utilización de sistemas institucionales de gestión de información curricular como la mejor herramienta para gestionar el conocimiento científico de una institución, así como la identificación e interpretación de patrones para la estructuración del conocimiento institucional. En este caso de estudio, se comprueba la validez del Sistema de Gestión de Información y Conocimiento Institucional (CV-UPR) como plataforma para obtener y contrastar información relevante sobre la estructuración del conocimiento proveniente delos resultados de las investigaciones, según las taxonomías establecidas, yel análisis de patrones por líneas investigativas y proyectos de investigación.

\section{Referencias bibliográficas}

Armas, D.; Díaz, M. y Giraldes, R. (2008), "Sistema Institucional para la Gestión de la Ciencia y la Técnica en Universidades: una perspectiva cienciométrica para su análisis y evaluación", en Memorias Información, 2008, La Habana, Cuba.

Báez, J. M.; Peset, F.; Núñez, F. y Ferrer, A. (2008), “CVN: normalización de los currículos científicos", en El profesional de la información, 17 (2), marzo-abril, 213-220.

Dahlberg, I. (2006), Knowledge Organization. Disponible en: http:// www.iva.dk/bh/lifeboat_ko/concepts/Knowledge_organization_ Dahlberg.htm [Fecha de consulta: 6 de febrero de 2010].

García, A. (2001), "Redes digitales y exomemoria", en Binaria: revista decomunicación, culturaytecnología, 1.Disponibleen:http://www. ic-journal.org/data/downloads/1265039267-2garcia-gutierrez.pdf [Fecha de consulta: 22 de febrero de 2009].

Gaughan, M.; Branco, P. y Bozeman, B. (2007), "Using quasi-experimental design and the curriculum vitae to evaluate impacts of earmarked center funding on faculty productivity, collaboration, and grant activity", en Proceedings of ISSI 2007: 11th International Conference of the InternationalSocietyforScientometricsandInformetrics, vols.I-II,305-313. Hjørland, B. (2003), "Fundamentals of knowledge organization", en Knowledge Organization, 30 (2), 87-111.

- (2005) (ed.), Lifeboat for knowledge organization. Disponible en: http://www.db.dk/bh/lifeboat_ko/home.htm [Fecha de consulta: 31 de mayo de 2009].

Hjørland, B. y Albrechtsen, H. (1995), "Toward a new horizon in information science: domain analysis", en Journal of the American Society for Information Science, 6 (6), 400-425.

Jaramillo, H.; Lopera, C. y Albán, M. C. (2008), Carreras Académicas. Utilización delCVparala modelación decarrerasacadémicasycientíficas, Bogotá: Facultad de Economía, Universidad del Rosario. Disponible en http://www.urosario.edu.co/FASE1/economia/do cumentos/pdf/bi96.pdf [Fecha de consulta: 16 de mayo de 2012]. 
Martín-Sempere, M. J. y Rey Rocha, J. (2009), El currículum vitae y la encuestacomofuentesdedatosparalaobtencióndeindicadoresde la actividad científica de los investigadores. Disponible en: http:// www.ricyt.edu.ar/interior/normalizacion/III_bib/Sempere_Rey. pdf [Fecha de consulta: 16 de abril de 2008].

Morin, E. (1995), "Sobre la interdisciplinariedad", en Revista Complejidad, 1 (0), 4-8. Disponible en: http://www.pensamientocomplejo. com.ar/docs/files/morinsobreinterdisciplinaridad.pdf [Fecha de consulta: 30 de febrero de 2010].

Navarrete, J. y Banqueri, J. (2008), “Los sistemas de información científica: herramientas para medir el impacto de la investigación biomédica", en Revista Medicina Clínica, 131, 71-80, Barcelona. (Supl. 5)

Peña, T. (2010), "El acceso al saber requiere aplicar organización y representación del conocimiento", en Revista Interamericana de Bibliotecología, 33 (1), enero-junio, 13-29. Universidad de Antioquia. Medellín, Colombia. Disponible en: http://redalyc.uaemex.mx/ src/inicio/ArtPdfRed.jsp?iCve=179015628001 [Fecha de consulta: 12 de enero de 2011].

Smiraglia, P. (2012), "Knowledge organization: some trends in an emergent domain", en El Profesional de la Información, 21 (3), mayo-junio, 225-227. Disponible en: http://dx.doi.org/10.3145/ epi.2012.may.01 [Fecha de consulta: 22 de septiembre de 2012].

Spinak, E. (1998), "Indicadores cienciométricos", en Ciência da Informação, 27 (2), 141-148.

Universidad de Salamanca (2005), Manual de Usuario Portal del Investigador. Disponible en: http://campus.usal.es/ infouxxi-inv/archi vos/manualuxxi.doc [Fecha de consulta: 25 de mayo de 2009].

Para citar este artículo como revista electrónica:

Rivero Amador, Soleidy et al. 2016. “Patrones para la organización del conocimiento en los Sistemas de Información Curricular. Un caso deestudio".InvestigaciónBibliotecológica:Archivonomía,Bibliotecologíaelnformación.68:91-107.Aquíseagregaladirecciónelectrónica (Consultado el día-mes-año)

Para citar este artículo tomado de un servicio de información:

Rivero Amador, Soleidy et al. 2016. "Patrones para la organización del conocimiento en los Sistemas de Información Curricular. Un caso de estudio".InvestigaciónBibliotecológica:Archivonomía,Bibliotecología elnformación.68:91-107.En:Aquíseagregaelnombredelserviciodeinformación y la dirección electrónica (Consultado el día-mes-año) 
